\title{
IMAGING
}

\section{MRI in the characterization and local staging of testicular neoplasms}

Tsili AC, Argyropoulou MI, Giannakis D, Sofikitis N, Tsampoulas K

Department of Clinical Radiology, University Hospital of Ioannina, Ioannina, Greece

AJR Am J Roentgenol. 2010 Mar;194(3):682-9.

Objective: The purpose of this study was to assess the role of MRI in the preoperative characterization and local staging of testicular neoplasms.

Subjects and Methods: MRI was performed on 33 patients referred because a testicular mass had been detected clinically and sonographically. Both T1- and T2-weighted sequences were performed with a 1.5-T MRI unit. Gadolinium chelate was administered IV in all cases. We recorded the presence of a lesion and whether the histologic diagnosis of testicular malignancy could have been predicted on the basis of MRI features. For testicular neoplasms, local extension of disease was studied. The MRI findings were correlated with the surgical and histopathologic results.

Results: Histologic examination revealed 36 intratesticular lesions, 28 (78\%) of which were malignant and eight benign. Thirteen malignant testicular tumors (46\%) were confined within the testis, $12(43 \%)$ had invaded the testicular tunicae or epididymis, and three (11\%) had invaded the spermatic cord. The sensitivity and specificity of MRI in differentiating benign from malignant intratesticular lesions were 100\% (95\% CI, 87.9-100\%) and $87.5 \%$ (95\% CI, 52.9-97.7\%). The rate of correspondence between MRI and histologic diagnosis in the local staging of testicular tumors was $92.8 \%(26 / 28)$.

Conclusion: MRI is a good diagnostic tool for the evaluation of testicular disease. It is highly accurate in the preoperative characterization and local staging of testicular neoplasms.

\section{Editorial Comment}

High-resolution sonography (US), with color or power Doppler has become the imaging modality of choice for the evaluation of scrotal abnormalities. US is an accurate method in distinguishing intratesticular from extratesticular lesions, a key point in the diagnostic evaluation of scrotal disease. Most intratesticular solid lesions are malignant, whereas extratesticular lesions are usually benign. Although sonography cannot accurately differentiate seminomatous from non-seminomatous tumors, their findings when combined with clinical information allow us to narrow the differential diagnosis of the majority of scrotal masses. Sonography can also be useful for local staging of testicular tumors, although it has limitation for the detection of the invasion of the spermatic cord (1). In such situation, very large scrotal mass or in inclusive sonographic studies, MRI should be performed as a complimentary tool.

The authors of this study nicely show that MRI is an efficient diagnostic tool to evaluate testicular masses and accurately differentiate between benign and malignant intratesticular tumors. With MRI, 87.5\% of benign intratesticular mass lesions were characterized correctly. The overall accuracy of MRI in estimating the local extent of malignant testicular tumors was $93 \%$. Contrary to US, MRI was adequate tool for the demonstration of invasion of the spermatic cord by the intratesticular tumor. Unfortunately, similarly to what happens with sonography, focal granulomatous orchitis may also simulate testicular tumor on MRI studies. The authors pointed out one major limitation of this study; they did not compare the diagnostic performances of sonography and MRI in the diagnosing and characterization of testicular disease. Although high-resolution sonography continues to be the imaging modality of choice, MRI is an efficient technique for testicular imaging. 


\section{Urological Survey}

\section{Reference}

1. Prando D: Contribution of Sonography in the Study of Testicular Tumor. PhD Thesis. Federal University of São Paulo, São Paulo, SP, Brazil. 1988

Dr. Adilson Prando

Head, Department of Radiology and Diagnostic Imaging, Vera Cruz Hospital Campinas, São Paulo, Brazil

E-mail: adilson.prando@gmail.com

doi: $10.1590 / S 1677-553820100003000019$

\section{Imaging of prostate cancer local recurrences: why and how?}

Rouvière $\mathrm{O}$, Vitry $\mathrm{T}$, Lyonnet $\mathrm{D}$

Université de Lyon, Lyon, France

Eur Radiol. 2010; 20: 1254-66.

Objective: Because prostate cancer local recurrences can be efficiently treated by salvage therapies, it becomes critical to detect them early.

Methods: The first alert is the rise of the prostate specific antigen (PSA) level after the post-treatment nadir, which can correspond to a distant recurrence, a local recurrence or both. This so-called biochemical failure (BF) is defined as PSA level $>0.2 \mathrm{ng} / \mathrm{ml}$ after radical prostatectomy (RP) and PSA level $>$ nadir $+2 \mathrm{ng} / \mathrm{ml}$ after radiotherapy. There is no consensual definition of BF after cryotherapy, high-intensity focused ultrasound (HIFU) ablation or brachytherapy.

Results: Local recurrences after RP are treated by radiotherapy, those after radiotherapy by RP, cryotherapy, brachytherapy or HIFU ablation. Recurrences after cryotherapy or HIFU ablation can be treated by a second session or radiotherapy. Recurrences after brachytherapy are difficult to treat. In patients with BF, MRI can detect local recurrences, whatever the initial treatment was. Dynamic contrast-enhanced MRI seems particularly accurate. The role of spectroscopy remains controversial. Ultrasound-based techniques are less accurate, but this may change with the advent of ultrasonic contrast media.

Conclusion: These recent advances in imaging may improve the outcome of salvage therapies (by improving patient selection and treatment targeting) and should open the way to focal salvage treatments in the near future.

\section{Editorial Comment}

The authors should be congratulated for reviewing this important issue on uro-oncology. Important aspects of local recurrence after radical prostatectomy (RP), external-beam radiotherapy (EBRT), HIFU ablation, cryotherapy and brachytherapy are presented and discussed. For each modality of local treatment of prostate cancer, the authors define biochemical failure and discuss treatment options and the role of imaging techniques for the detection of tumor recurrence.

In our experience, dynamic-contrast enhanced MRI is the best modality for the detection of local recurrence after RP. For local recurrence after EBRT our better results are obtained with spectroscopy although dynamic-contrast enhanced MRI can also be useful in most cases. We also prefer to use spectroscopy for the detection of local tumor recurrence after brachytherapy. The quality of dynamic-contrast enhanced MRI stud- 